\title{
Effect of Ti on microstructure and properties of aluminum sacrificial anode containing rare earth elements
}

\author{
Cheng,kun ${ }^{1, a}$, Liu, xin ${ }^{1, b}$, Xie,guangwen $^{1}$, Duan jizhou ${ }^{2}$, Zhang jie ${ }^{2}$ \\ ${ }^{1}$ College of Materials Science and Engineering, Qingdao University of Science \& Technology, \\ Qingdao 266042, China; \\ ${ }^{2}$ Key Laboratory of Marine Environmental Corrosion and Bio-fouling, Institute of \\ Oceanology, Chinese Academy of Sciences, Qingdao, 266071, China \\ a1573117092@qq.com, bxliu_neu@126.com
}

Keywords: Al sacrificial anodes, Ti, microstructure, electrochemical performance.

\begin{abstract}
The optimum amount of Ti element in aluminum alloy sacrificial anodes was explored by analyzing microstructure, electrochemical impedance and polarization curve of the anode. And their corrosion behavior was determined preliminarily. The results showed that the aluminum anodes with $0.1 \%$ Ti have fine grains, dissolve uniformly, and have good activation performance. The reason is that the addition of titanium can make the alloy elements dissolved uniformly, reduce the intergranular corrosion.
\end{abstract}

\section{Introduction}

The corrosion and protection of metal materials is an important engineering problem. Due to its convenience, simplicity and significant effect of Sacrificial anode cathodic protection that is widely used $^{[1,2]}$. Currently, the sacrificial anode materials include aluminum alloys, zinc-based alloys and magnesium-based alloys ${ }^{[3,4,5]}$. The aluminum alloy with high current efficiency, high capacity, light weight and low price ${ }^{[6,7,8]}$ is widely used in the cathodic protection system for the steel material.

It is reported that the performance of the aluminum anode can be improved by adding Ti element on the basis of Al-Zn-In-Si-Ce. The addition of $\mathrm{Ti}$ element is appropriate to reduce the working potential and stable potential of the aluminum anode ${ }^{[9,10]}$, make the corrosion of aluminum anode more uniformly and in a certain extent, it improves the current efficiency of the alloy and improves the capacity of the power, as well as reduces intergranular corrosion and casting hot crack phenomenon.

\section{Materials and methods}

Tab. 1 chemical composition of Al-Zn-In-Si based alloy sacrificial anode (\%wt)

\begin{tabular}{ccccccc}
\hline $\begin{array}{c}\text { Anode } \\
\text { name }\end{array}$ & Zn & In & Si & Ce & Ti & Al \\
\hline 1 & 5.81 & 0.028 & 0.12 & 0.1 & 0.03 & \\
2 & 5.81 & 0.028 & 0.12 & 0.1 & 0.05 & Allowance \\
3 & 5.81 & 0.028 & 0.12 & 0.1 & 0.07 & \\
4 & 5.81 & 0.028 & 0.12 & 0.1 & 0.1 & \\
\hline
\end{tabular}

According to the above ratio of elements, the aluminum alloy samples were cast at $750{ }^{\circ} \mathrm{C}$, and machined to $\phi 16 \times 48 \mathrm{~mm}^{2}$ and set aside. Firstly, the appearance of aluminum polished before and after corrosion was observed in the optical microscope. Then, the system with three electrodes was used to test the polarization curve and electrochemical impedance in $3 \% \mathrm{wt} \mathrm{NaCl}$ solution. 


\section{Results and Discussion}

\subsection{Microstructure Analysis}
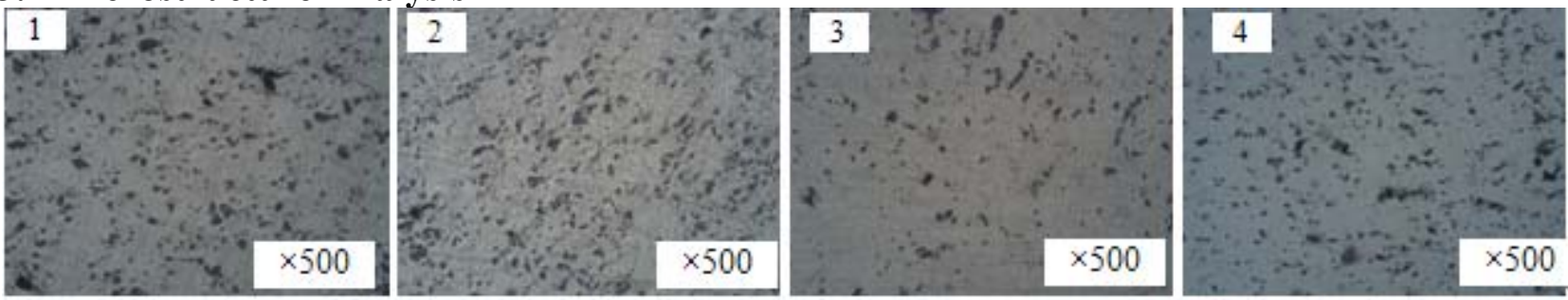

Fig.1 Microstructure of Al alloy anodes before corrosion
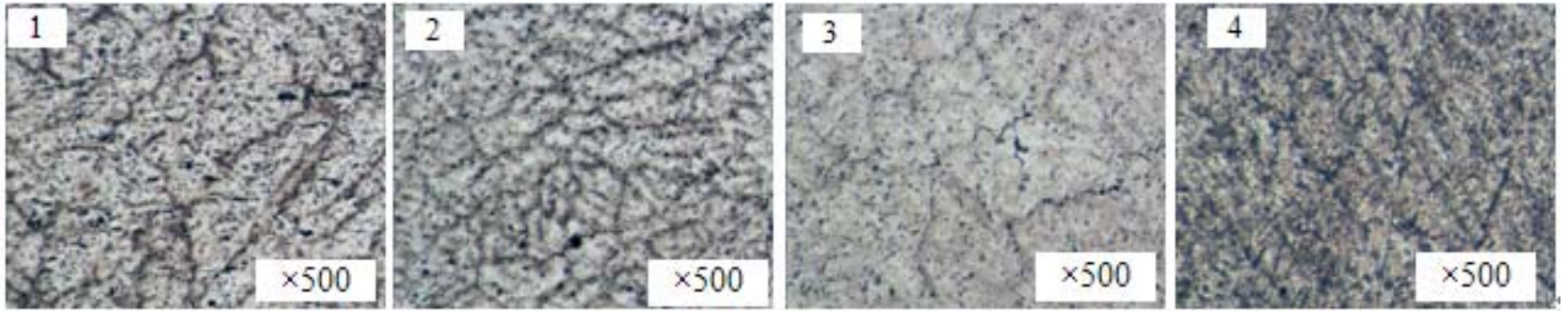

Fig.2 Microstructure of Al alloy anodes after corrosion

The microstructure of Al alloy anodes before corrosion generally can be observed. The number of precipitates decreases firstly and then increases. Because titanium element can cause the distribution of zinc and decrease the segregation of zinc and indium. When the adding amount of Ti exceeds a certain value, titanium element also could be the second phase precipitation. From the microstructure, the grain boundary precipitates decrease with the increase of Ti content after corrosion. The reason is that the Ti element makes the zinc and indium more dissolved in the crystal and reduces the intergranular corrosion.

\subsection{Potentiodynamic polarization Analysis}

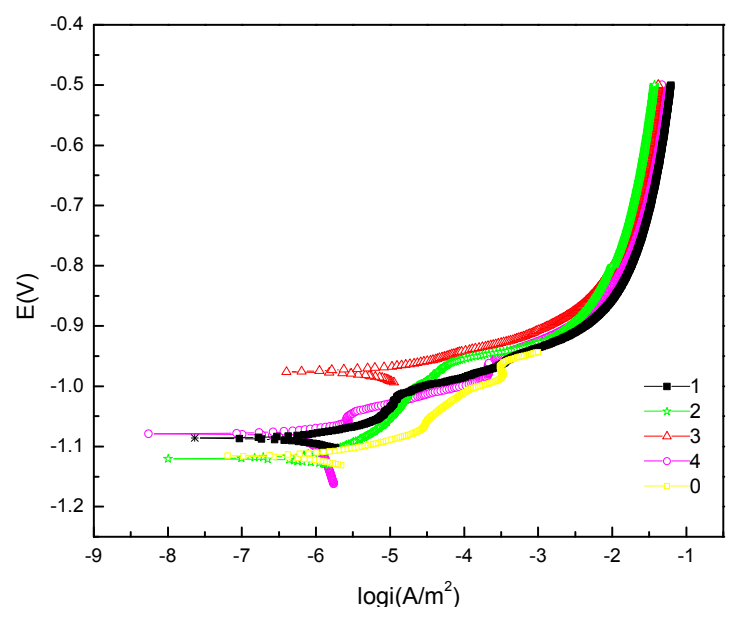

Fig.3 Anodic polarization curve of Al alloy anodes

The protection potential of steel structure in seawater is $-0.8 \mathrm{~V}(\mathrm{Vs}$. SCE), which is generally required the driving potential with $0.25 \mathrm{~V}$, that is, the anode is between $-1.1 \sim-1.0 \mathrm{~V}$ (Vs. SCE) when the steel structures can be effectively protected. From fig. 3 it can be seen that the corrosion potential of sample 1, 3 and 4, with the addition of Ti element, appears different degree of positive shift. The potential of sample 1 and 4 respectively with $0.03 \%$ and $0.1 \%$ Ti content components is held at -1.0 $\sim 1.1 \mathrm{~V}$ (Vs. SCE). 


\subsection{Electrochemical Impedance Analysis}
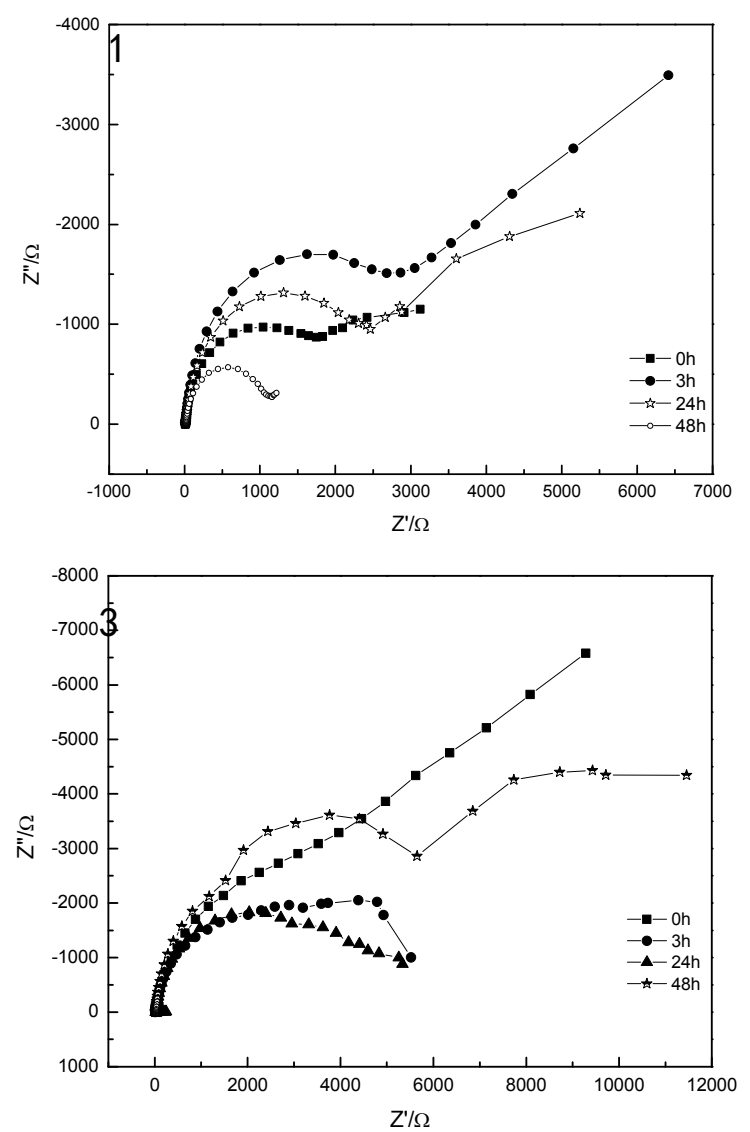
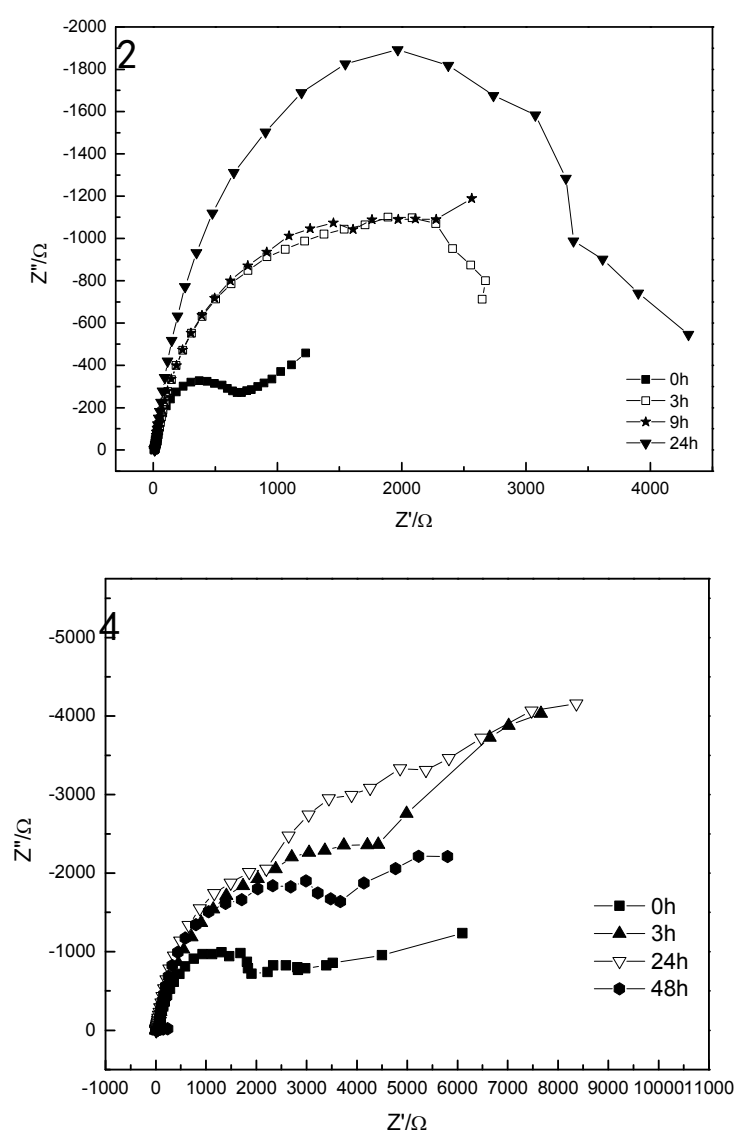

Fig.4 EIS of Al alloy anodes

The fig. 4-1 indicates that the electrochemical impedance spectroscopy shape of samples is similar in both groups consisting of a capacitive arc and a short line segments. It is obviously that the pitting reactions taken place. The capacitive arc and short straight line increase firstly and then decrease that show the reaction is blocked and the charges transfer are difficulty. Later, with the deepening of the oxide film damage, the corrosion products draw off and the anode dissolution rate increase. The capacitive arc decreases, line segment of length decreases gradually. Fig 4-4 is similar to Fig.4-1. The principle and process of corrosion are as above.

It can be seen from Fig4-2, the impedance spectroscopy are a capacitive loop and a straight line due to dispersion effects at $0 \mathrm{~h}$ leading to the arc anode capacitance being not standards semicircle, which shows the state of the electrode surface occurs changes and pitting appear. With the extension of soaking time, the anode capacitance arc of $2 \mathrm{~h}, 9 \mathrm{~h}$ ant $24 \mathrm{~h}$ become larger gradually, indicating that the anodic charge transfer resistance greater, the degree of reaction disruption increases. The main reason may be that the corrosion products don't spread to the solution and attach on the surface of the anode, the continuing dissolution of the anode is effected.

In the Fig 4-3, in the low-frequency part the impedance spectrum shows a 45 degree diagonal at 0 $\mathrm{h}$. With immersion time increasing, the capacitance arc decreases gradually. At $48 \mathrm{~h}$ the impedance spectrum begins to appear a short straight line. This shows that at the beginning of anode corrosion, the anode surface have a thick and dense passivation membrane. Since the membrane resistance is large, the migration of ions is suppressed greatly. With the increase of immersion time, the reaction degree is decreased, the film becomes thinner, and it may be in the pitting induction period. When the immersion time increases, the alloy is in the pitting stage. 


\section{Summary}

1. By the observation of microstructure, it can be seen that with the increase of Ti content, the segregation decreases and the alloy elements dissolve more evenly.

2 . Through the polarization curve analysis, the aluminum anode curve with $0.1 \% \mathrm{Ti}$ content is smooth, and its corrosion potential is between $-1.1 \sim-1.0 \mathrm{~V}(\mathrm{Vs}$. SHE) as well as the activation performance is good.

3. From electrochemical impedance spectroscopy, aluminum anodes that contain $0.03 \%, 0.07 \%$ and $0.1 \%$ of $\mathrm{Ti}$ are uniformly dissolved.

In summary, the $\mathrm{Al}$ anode containing $0.1 \% \mathrm{Ti}$ has the best comprehensive performance. And the best addition amount of $\mathrm{Ti}$ in the Al-Zn-In-Si-Ce-Ti aluminum anode is determined.

\section{Acknowledgements}

This work was financially supported by Open Subject of Key Laboratory of Marine Environmental Corrosion and Bio-fouling, Institute of Oceanology, Chinese Academy of Sciences( MCKF201407).

\section{References}

[1] Weng Songgan, Du Min, Huang Zhiqiang, etc. The effect of the alloy composition on the Al-Zn-In-Mg-Ti sacrifice anode [J]. China Ocean University: Natural Science Edition, 2012.

[2] Song Yuehai. Research of high performance aluminum, zinc, magnesium alloy series sacrificial anode materials [D]. Kunming University of Science and Technology, 2003.

[3] Bai Yun. The metallurgical studies of Al-Zn-In-Si-Mg alloy sacrificial anodes [J] Anshan Normal University, 1999, (3): 94-98.

[4] Chenmeng Li. anode development and application of marine structure magnesium aluminum composite sacrifice[D] Chongqing University, 2009.

[5] $\mathrm{Hu}$ Shengnan. Under simulated deep ocean environment research performance of Al-Zn-In sacrificial anode [D] Harbin Engineering University, 2012.

[6] Mayumi K, Mayumi K. Design Procedure of Aluminum Alloy Anodes for Cathodic Protection of Steel Structure in Seawater[J]. Zairyo-to-Kankyo, 2008, 57(8).

[7] Li Qing, Yin Yanxi, Jiang Honglin, electrochemical properties of high active aluminum alloy anode material [J] Materials Protection, 2008, 41 (10): 20-22.

[8] $\mathrm{Xu}$ Feng. Preparation and performance of aluminum alloys sacrificial anode [D].Nanjing University of Technology, 2004.

[9] Jiao Mengwang. Study on the microstructure and electrochemical properties of Al-Zn-In-Mg-Ti sacrificial anode material [D]. Henan University of Science and Technology, 2008.

[10] Zhu Chengde,Li Yi. The research of a new type of highly efficient sacrificial anode. material protection, 1997, (8): 21-23. 